\title{
A Clinical and Electrophysiological Study of Peripheral Neuropathies in Predialysis Chronic Kidney Disease Patients and Relation of Severity of Peripheral Neuropathy with Degree of Renal Failure
}

\author{
Dushyanth Babu Jasti, Sarat Mallipeddi, A. Apparao, B. Vengamma, V. Sivakumarl, Satyarao Kolli
}

Departments of Neurology and ${ }^{~}$ Nephrology, SVIMS, Tirupati, Andhra Pradesh, India
Objective: To study the prevalence, clinical features, electrophysiological features, and severity of peripheral neuropathy in predialysis chronic kidney disease (CKD) patients with respect to severity of renal failure and presence of diabetes mellitus. Materials and Methods: Between May 2015 and December 2016, 200 predialysis CKD patients were assessed prospectively. Results: The prevalence of peripheral neuropathy in predialysis CKD patients in the present study was $45 \%$ based on clinical symptoms and 90\% electrophysiologically. Mean age of 200 predialysis CKD patients who participated in the study was $53.2 \pm 13.2$ years. One hundred and thirty-six $(68 \%)$ patients were male and $64(32 \%)$ patients were female. Mean duration of disease was $2.2 \pm 1.6$ years. Nearly $45 \%$ patients of patients had asymptomatic peripheral neuropathy in the present study, which was more common in mild-to-moderate renal failure group. One hundred twenty-six patients $(63 \%)$ had definite damage and 54 patients $(27 \%)$ had early damage. In mild-to-moderate renal failure $(n=100)$ and severe renal failure patients $(n=100)$, $88 \%$ and $92 \%$ had significant peripheral neuropathy, respectively. Most common nerves involved were sural nerve, median sensory nerve, and ulnar sensory nerve. Diabetic patients (97\%) showed more severe and high prevalence of peripheral neuropathy when compared to nondiabetic patients $(83 \%)$. Most common patterns were pure axonal sensorimotor neuropathy and mixed sensorimotor neuropathy. Conclusion: Peripheral neuropathy is common in predialysis patients, prevalence and severity of which increases as renal failure worsens. Predialysis patients with diabetes show higher prevalence and severity of peripheral neuropathy when compared with nondiabetics.

KEYWORDS: Chronic kidney disease, diabetes mellitus, nerve conduction studies, peripheral neuropathy, predialysis

\section{INTRODUCTION}

C hronic kidney disease ${ }^{[1-3]}$ (CKD) is defined as the kidney damage lasting more than 3 months, which is characterized by structural or functional abnormalities of the kidney, with or without decreased glomerular filtration rate. Organic deteriorated products which are generally cleared by kidneys accumulate in CKD which leads to uremic disease. ${ }^{[4]}$ Approximately $60 \%$ of the population with CKD will encounter neurological complications, ${ }^{[5]}$ which affects at all levels of nervous system, peripheral and central, resulting in altered mental state, continued disability and

\begin{tabular}{|l|l|}
\hline \multicolumn{2}{|c|}{ Access this article online } \\
\hline Quick Response Code: & Website: \\
\hline
\end{tabular}

weakness. ${ }^{[6]}$ Uremic neuropathy is one of the most common neurological complications of uremia. Distal symmetrical sensorimotor peripheral neuropathy is the most common pattern of neuropathy in $\mathrm{CKD}$, and it predominantly affects lower limbs when compared to upper limbs.

Address for correspondence: Dr. Dushyanth Babu Jasti, Department of Neurology, SVIMS, Alipiri Road, Tirupati, Andhra Pradesh, India. E-mail: dushyanthjasti@yahoo.co.in

This is an open access article distributed under the terms of the Creative Commons Attribution-NonCommercial-ShareAlike 3.0 License, which allows others to remix, tweak, and build upon the work non-commercially, as long as the author is credited and the new creations are licensed under the identical terms.

For reprints contact: reprints@medknow.com

How to cite this article: Jasti DB, Mallipeddi S, Apparao A, Vengamma B, Sivakumar V, Kolli S. A clinical and electrophysiological study of peripheral neuropathies in predialysis chronic kidney disease patients and relation of severity of peripheral neuropathy with degree of renal failure. J Neurosci Rural Pract 2017;8:516-24. 
Uremic neuropathy was first reported by Kussmaul in 1863. Asbury et al. ${ }^{[7]}$ gave detailed explanation regarding clinicopathological features of uremic neuropathy. Dyck et al. in 1971 established the present concept of uremic neuropathy which was based on nerve conduction studies (NCSs), electron microscopy, and light microscopy studies. ${ }^{[8]}$ The slowing of nerve conduction in the clinically affected segments was demonstrated in an article by Nielsen and Winkel and Bolton et al. in 1970, ${ }^{[9,10]}$ which showed a negative correlation with degree of renal failure and speed of nerve conduction. According to Nielsen, $77 \%$ of CKD patients had symptoms of peripheral neuropathy, while $51 \%$ had signs of peripheral neuropathy. ${ }^{[1]}$

Uremic neuropathy usually involves large diameter axons. ${ }^{[11]}$ Uremic neuropathy is often multifactorial, which is exacerbated by nutritional deficiency, hypocalcemia, and hypomagnesemia. Uremic toxins such as guanidine compounds, polyamines, phenol metabolites, myoinositol, and 3-carboxy-4-methyl-5-propyl-2-fluranpropanoic acid were proposed as causative agents of uremic neuropathy. ${ }^{[12]}$ Uremic toxins may enter the endoneurium space and cause direct nerve damage due to water and electrolyte shifts with expansion or retraction of space. Middle molecules which are markedly elevated in CKD patients are one of the proposed causative agents of uremic neuropathy. ${ }^{[13]}$ Despite recent therapeutic advances, uremic neuropathy often fails to respond completely to available treatment modalities. Even though peripheral neuropathy is a well-described neurological complication in end-stage renal disease patients, there are no major Indian studies addressing this issue. In predialysis patients, peripheral neuropathy is expected to worsen with worsening renal parameters. However, data regarding clinical and electrophysiological features of peripheral neuropathy in CKD patients who are not yet initiated on dialysis are sparse in South India. The present study was done to assess the prevalence and clinical and electrophysiological features of peripheral neuropathy in predialysis patients and effect of diabetes mellitus on peripheral neuropathy.

\section{Materials and Methods}

During May 2015 to December 2016, 200 consecutive patients diagnosed to have and treated for CKD with serum creatinine $>2 \mathrm{mg} / \mathrm{dl}$ (predialysis patients) at Sri Venkateswara Institute of Medical Sciences, Tirupati, were included in the present study. Patients with preexisting peripheral neuropathy before the diagnosis of CKD and CKD patients who were initiated on dialysis were excluded from the present study. The study was approved by the institutional ethics committee, and written informed consent was obtained from the patients for their participation in the study.

\section{Study procedure}

Two hundred consecutive CKD patients with serum creatinine $>2 \mathrm{mg} / \mathrm{dl}$ who had given consent to participate in the study were divided into two groups. Group 1 included 100 predialysis CKD patients with serum creatinine between 2 and $4.9 \mathrm{mg} / \mathrm{dL}$ (mild-to-moderate renal failure). Group 2 included 100 predialysis patients with serum creatinine $\geq 5 \mathrm{mg} / \mathrm{dl}$ (severe renal failure). Each group was further subdivided into two subgroups: each subgroup included 50 diabetic patients and 50 nondiabetic patients.

Detailed history was elicited pertaining to symptoms of peripheral neuropathy and diabetes mellitus. Detailed general physical examination and neurological examination were done and documented. Biochemical investigations including blood urea, serum creatinine, and blood sugars were measured in all the patients as per the standard methods used in the department of biochemistry.

\section{Nerve conduction studies}

All cases were subjected to NCSs using Medelec Synergy and Natus machines. NCS procedure was done for both motor conductions and sensory conductions. Median nerve, ulnar nerve, common peroneal nerve, and posterior tibial nerve were assessed for motor conductions. Median nerve, ulnar nerve, and sural nerve were assessed for sensory conductions. In motor conductions, distal latency, conduction velocity, amplitude, and F wave were assessed. In sensory conductions, distal latency, conduction velocity, and amplitude were assessed.

\section{Motor nerve conduction studies procedure}

The gain was normally set at $2-5 \mathrm{mV}$ per division for the motor conduction studies. ${ }^{[14]}$ The recording electrodes were placed on the muscle being studied. The belly-tendon montage was used commonly. The center of the muscle belly (over the motor endplate) was used for placing the active recording electrode (also known as G1) and the reference electrode (also known as G2) was placed distally, over the tendon of the muscle. The nerve that supplies the muscle was used for placing the stimulator, where the cathode was placed close to the recording electrode. The duration of the electrical pulse was generally set to $200 \mathrm{~ms}$ for the motor NCSs. To achieve supramaximal stimulation, current in the range of $20-50 \mathrm{~mA}$ was used. The underlying nerve fibers were brought to action potential as the current was steadily increased from a baseline, usually by 5-10 mA. 
The summation of all the underlying individual muscle fiber action potentials was represented by the compound muscle action potential (CMAP). When all the nerve fibers have been excited and the supramaximal stimulation has been achieved, the CMAP will no longer increase in size.

For median nerve motor conduction studies, the recording electrode was placed over the motor point of the abductor pollicis brevis muscle, at the midpoint of a line drawn from the first metacarpophalangeal joint to the insertion of the tendon of the flexor carpi radialis muscle, and the reference electrode was placed over the distal interphalangeal joint. Midarm, antecubital fossa, and wrist were sites of stimulation for median nerve motor conduction studies. For ulnar nerve motor conduction studies, the recording electrode was placed over the motor point of the abductor digiti minimi muscle, at the midpoint of a line between the fifth metacarpophalangeal joint and the pisiform bone, with the reference electrode over the middle phalanx of digit V. Axilla, above elbow, ulnar groove, and medial wrist were sites of stimulation for ulnar nerve motor conduction studies. For the posterior tibial nerve, the CMAP was recorded by placing the active electrode over the middle of the adductor hallucis muscle, and the reference electrode over the proximal phalanx of digit I. The posterior tibial nerve was stimulated below the medial malleolus and in the popliteal fossa. For common peroneal nerve motor conduction studies, the recording electrode was placed in the middle of the extensor digitorum brevis muscle. The common peroneal nerve was stimulated at the ankle, $80 \mathrm{~mm}$ proximal to the recording electrode, lateral to the tendon of tibialis anterior muscle, and below the knee $20-50 \mathrm{~mm}$ distal to the proximal part of the caput fibula.

Latency was described as the time from the stimulus to the initial CMAP deflection from the baseline. The CMAP amplitude was measured from the baseline to the negative peak. Conduction velocity was calculated using the formula as follows: Distance between the proximal and distal stimulation sites/proximal latency - distal latency. The standardized normal adult values of motor NCSs in both upper and lower extremities as per our electrophysiology laboratory are shown in Table $1 .^{[15]}$

The $\mathrm{F}$ response also known as the late motor response occurs after the CMAP. ${ }^{[16]}$ Normal minimal F latency was $25-30 \mathrm{~ms}$ in median and ulnar nerves, while it was 45-59 ms in common peroneal and posterior tibial nerves.

\section{Sensory conduction studies}

Median and ulnar sensory nerve action potentials (SNAPs) were obtained orthodromically, stimulating from the index finger (median nerve) or the little finger (ulnar nerve) and recording at the wrist. Sural SNAPs were obtained antidromically, recording behind the lateral malleolus and stimulating on the dorsal aspect of the calf, $140 \mathrm{~mm}$ proximal to the recording site. The responses were averaged at least 10 times. The standardized normal adult values of sensory NCSs in both upper and lower extremities as per our electrophysiology laboratory are shown in Table 2.[17]

Based on electrophysiological parameters, peripheral neuropathy patterns were subclassified into axonal neuropathy, demyelinating neuropathy, and mixed neuropathy. In axonal neuropathy, CMAPs decrease, conduction velocities are normal or slightly decreased but never $475 \%$ of the lower limit of normal, and distal latencies are normal or slightly prolonged but never $>130 \%$ of the upper limit of normal. In demyelinating neuropathy, CMAPs are usually normal with marked slowing of conduction velocity (slower than $75 \%$ of the lower limit of normal) and/or marked prolongation of distal latency (longer than 130\% of the upper limit of normal). It was classified as mixed neuropathy if it has features of both axonal neuropathy and demyelinating neuropathy. Degree of severity of peripheral neuropathy was divided into three groups: normal, early damage, and definite damage, according to the number of peripheral nerves involved. Normal or no peripheral damage was defined if NCSs were normal or only one peripheral nerve was involved. Early damage, if two or three peripheral nerves were involved, and definite damage, if more than three peripheral nerves were involved.

Table 1: Motor nerve conduction studies: Standardized normal adult values in both upper and lower extremities

\begin{tabular}{llccc}
\hline Nerve & Record & Amplitude $(\mathbf{m V})$ & $\mathbf{C V}(\mathbf{m} / \mathbf{s})$ & dL $(\mathbf{m s})$ \\
\hline Median & APB & $>6$ & $>49$ & $<4.4$ \\
Ulnar & ADM & $>6$ & $>49$ & $<3.3$ \\
CP & EDB & $>4$ & $>44$ & $<4.5$ \\
Posterior tibial & AH & $>5$ & $>41$ & $<4.5$ \\
\hline
\end{tabular}

APB: Abductor pollicis brevis, ADM: Abductor digiti minimi, EDB: Extensor digitorum brevis, AH: Abductor halluces, CV: Conduction velocity, dL: Distal latency, CP: Common peroneal

Table 2: Sensory nerve conduction studies: standardized normal adult values in both upper and lower extremities

\begin{tabular}{llccc}
\hline Nerve & $\begin{array}{l}\text { Recording } \\
\text { site }\end{array}$ & Amplitude (mV) & $\mathbf{C V}(\mathbf{m} / \mathbf{s})$ & $\begin{array}{c}\text { Distal peak } \\
\text { latency (ms) }\end{array}$ \\
\hline Median & Wrist & $>10$ & $>50$ & $<3.5$ \\
Ulnar & Wrist & $>10$ & $>50$ & $<2.5$ \\
Sural & Ankle & $>10$ & $>45$ & $<3.0$ \\
\hline
\end{tabular}

CV: Conduction velocity 


\section{Statistical analysis}

The data were collected and tabulated using Microsoft excel 2010 version. Data were analyzed using SPSS version 20. All the continuous variables were expressed as mean \pm standard deviation (SD) or median with interquartile range as appropriate. All categorical variables were expressed as frequencies (percentage). Independent $t$-test and ANOVA test were applied to compare nominal data between the groups and $P<0.05$ was considered statistically significant.

\section{RESULTS}

Between May 2015 and December 2016, 200 consecutive CKD patients with serum creatinine $>2 \mathrm{mg} / \mathrm{dl}$ who consented to participate were included in the study. The study plan is shown in Figure 1.

\section{Demographic characteristics}

The mean age in the present study was $53.2 \pm 13.2$ years. Males $(n=136,68 \%)$ outnumbered females $(n=64$, $32 \%)$. Mean duration of CKD was $2.2 \pm 1.6$ years. The mean serum creatinine and blood urea of 200 predialysis patients were $4.9 \pm 2.6 \mathrm{mg} / \mathrm{dl}$ and $95.7 \pm 53.2 \mathrm{mg} / \mathrm{dl}$, respectively. The prevalence of peripheral neuropathy in the present study was $45 \%$ based on clinical symptoms and $90 \%$ based on electrophysiological parameters.

The mean duration of diabetes mellitus in diabetic subgroups $(n=100)$ was $7.3 \pm 2.8$ years.

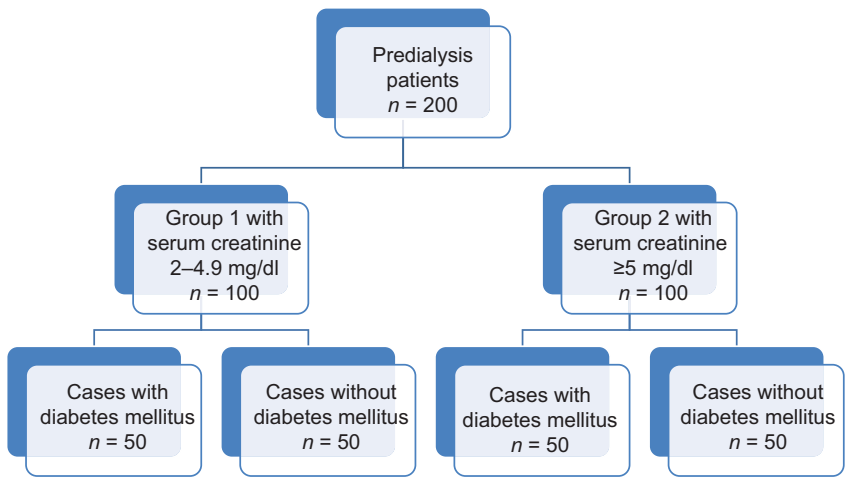

Figure 1: Study plan
Symptoms and signs of 200 predialysis patients are shown in Table 3. On comparing symptoms and signs of two groups of predialysis patients, patients with severe renal failure (Group 2) showed statistically significant positive symptoms $(P<0.001)$, absent ankle jerk $(P=0.023)$, impaired pain and temperature sensation $(P=0.048)$, and impaired vibration and joint position sense $(P=0.034)$.

\section{Electrophysiological parameters of 200 predialysis patients}

Nerve conduction abnormalities in 200 predialysis patients are shown in Table 4. Comparison of electrophysiological parameters of 200 predialysis patients, 100 patients with serum creatinine of 2-4.9 $\mathrm{mg} / \mathrm{dl}$ (Group 1), and 100 patients with serum creatinine of $\geq 5 \mathrm{mg} / \mathrm{dl}$ (Group 2) is shown in Table 5 . Among 200 predialysis CKD patients, 126 patients (63\%) had definite damage and 54 patients (27\%) had early damage. The most common patterns of peripheral neuropathy in 200 predialysis patients were pure axonal sensorimotor neuropathy pattern $(53 \%)$ followed by mixed sensorimotor neuropathy pattern (30\%).

Predialysis patients with mild-to-moderate renal failure (Group 1)

Mean age was $57.4 \pm 12.9$ years. Males $(n=70,70 \%)$ outnumbered females $(n=30,30 \%)$. Mean serum creatinine and blood urea in Group 1 were $2.7 \pm 0.6 \mathrm{mg} / \mathrm{dl}$ and $67.4 \pm 35.3 \mathrm{mg} / \mathrm{dl}$, respectively. The prevalence of peripheral neuropathy was 30\% based on clinical symptoms and $88 \%$ based on electrophysiological parameters.

Among 100 patients in Group 1, 54 patients (54\%) had definite damage, 34 patients (34\%) had early damage, and 12 patients $(12 \%)$ had no evidence of significant peripheral neuropathy. In diabetic subgroup $(n=50)$, definite damage was seen in $36(72 \%)$ patients and early damage was seen in $14(28 \%)$ patients. In nondiabetic subgroup $(n=50)$, definite damage was seen in $18(36 \%)$ patients, early damage was seen in $20(40 \%)$

\begin{tabular}{|c|c|c|c|c|}
\hline \multirow[t]{2}{*}{ Symptoms and signs } & \multirow{2}{*}{$\begin{array}{l}\text { Predialysis patients } \\
\quad(n=200), n(\%)\end{array}$} & \multicolumn{2}{|c|}{ Serum creatinine } & \multirow[t]{2}{*}{$P$} \\
\hline & & $2-4.9 \mathrm{mg} / \mathrm{dl}(n=100), n(\%)$ & $\geq 5 \mathrm{mg} / \mathrm{dl}(n=100), n(\%)$ & \\
\hline Motor weakness & 0 & 0 & 0 & NA \\
\hline Positive symptoms & $80(40)$ & $20(20)$ & $60(60)$ & $<0.001$ \\
\hline Negative symptoms & $70(35)$ & $30(30)$ & $40(40)$ & 0.402 \\
\hline Autonomic symptoms & $8(4)$ & 0 & $8(8)$ & 0.117 \\
\hline Wasting of limbs & 0 & 0 & 0 & NA \\
\hline Absent ankle jerk & $160(80)$ & $70(70)$ & $90(90)$ & 0.023 \\
\hline Impaired pain and temperature sensation & $42(21)$ & $12(12)$ & $30(30)$ & 0.048 \\
\hline Impaired vibration and joint position sense & $50(25)$ & $14(14)$ & $36(36)$ & 0.034 \\
\hline
\end{tabular}

NA: Not applicable 


\begin{tabular}{|c|c|c|c|}
\hline \multirow{2}{*}{$\begin{array}{l}\text { Nerve conduction } \\
\text { parameter }\end{array}$} & \multirow{2}{*}{$\begin{array}{l}\text { Predialysis patients } \\
(n=200), n(\%)\end{array}$} & \multicolumn{2}{|c|}{ Serum creatinine } \\
\hline & & $2-4.9 \mathrm{mg} / \mathrm{dl}(n=100), n(\%)$ & $\geq 5 \mathrm{mg} / \mathrm{dl}(n=100), n(\%)$ \\
\hline \multicolumn{4}{|l|}{ Median nerve } \\
\hline Prolonged mdL & $30 / 200(15)$ & $12 / 100(12)$ & 18/100 (18) \\
\hline Reduced CMAP & $60 / 200(30)$ & $16 / 100(16)$ & $44 / 100(44)$ \\
\hline Reduced MCV & $76 / 200(38)$ & $42 / 100(42)$ & $34 / 100(34)$ \\
\hline \multicolumn{4}{|l|}{ Ulnar nerve } \\
\hline Prolonged mdL & $24 / 200(12)$ & $8 / 100(8)$ & $16 / 100(16)$ \\
\hline Reduced CMAP & $60 / 200(30)$ & $28 / 100(28)$ & $32 / 100(32)$ \\
\hline Reduced MCV & $46 / 200(23)$ & $20 / 100(20)$ & $26 / 100(26)$ \\
\hline \multicolumn{4}{|l|}{ CP nerve } \\
\hline Prolonged mdL & $30 / 200(15)$ & $12 / 100(12)$ & $18 / 100(18)$ \\
\hline Reduced CMAP & $130 / 200(65)$ & $66 / 100(66)$ & $64 / 100(64)$ \\
\hline Reduced MCV & $104 / 200(52)$ & $48 / 100(48)$ & $56 / 100(56)$ \\
\hline \multicolumn{4}{|l|}{ Posterior tibial nerve } \\
\hline Prolonged mdL & $38 / 200(19)$ & $18 / 100(18)$ & $20 / 100(20)$ \\
\hline Reduced CMAP & $92 / 200(46)$ & $40 / 100(40)$ & $52 / 100(52)$ \\
\hline Reduced MCV & $86 / 200(43)$ & $44 / 100(44)$ & $42 / 100(42)$ \\
\hline \multicolumn{4}{|c|}{ Median nerve (sensory) } \\
\hline Reduced SNAP & $102 / 200(51)$ & $40 / 100(40)$ & $62 / 100(62)$ \\
\hline Reduced SCV & $132 / 200(66)$ & $58 / 100(58)$ & $74 / 100(74)$ \\
\hline \multicolumn{4}{|c|}{ Ulnar nerve (sensory) } \\
\hline Reduced SNAP & $124 / 200(62)$ & $46 / 100(46)$ & $78 / 100(78)$ \\
\hline Reduced SCV & $128 / 200(64)$ & $50 / 100(50)$ & $78 / 100(78)$ \\
\hline \multicolumn{4}{|l|}{ Sural nerve } \\
\hline Reduced SNAP & $166 / 200(83)$ & $86 / 100(86)$ & $80 / 100(80)$ \\
\hline Reduced SCV & $102 / 200(51)$ & $48 / 100(48)$ & $54 / 100(54)$ \\
\hline
\end{tabular}

patients, and $12(24 \%)$ patients had no significant peripheral neuropathy. The most common patterns of peripheral neuropathy were pure axonal sensorimotor neuropathy $(44 \%)$ followed by mixed sensorimotor neuropathy pattern $(40 \%)$.

On comparing symptoms and signs of two subgroups of Group 1, diabetic subgroup showed statistically significant absent ankle jerk $(P=0.012)$.

On comparing electrophysiological data between two subgroups of Group 1, diabetic subgroup showed statistically significant prolonged median nerve motor distal latency $(P<0.001)$, low median nerve motor conduction velocity $(P=0.001)$, prolonged median nerve $\mathrm{F}$ wave $(P=0.006)$, low ulnar nerve motor conduction velocity $(P<0.001)$, low ulnar nerve motor amplitude $(P=0.003)$, prolonged ulnar nerve F wave $(P<0.001)$, low common peroneal nerve motor conduction velocity $(P<0.001)$, low common peroneal nerve motor amplitude $(P=0.008)$, prolonged posterior tibial nerve motor distal latency $(P=0.044)$, low posterior tibial nerve motor conduction velocity $(P<0.001)$, low posterior tibial nerve motor amplitude $(P<0.007)$, prolonged posterior tibial $\mathrm{F}$ wave $(P=0.002)$, low median nerve sensory conduction velocity $(P=0.031)$, low ulnar nerve sensory conduction velocity $(P=0.025)$, low ulnar nerve sensory amplitude $(P=0.001)$, low sural nerve sensory conduction velocity $(P=0.021)$, and low sural nerve sensory amplitude $(P=0.004)$. Patients without diabetes mellitus showed statistically significant prolonged ulnar nerve motor distal latency $(P=0.020)$.

\section{Predialysis patients with severe renal failure (Group 2)}

Mean age was $49.0 \pm 12.3$ years. Males ( $n=66,66 \%$ ) outnumbered females ( $n=34,34 \%$ ). Mean serum creatinine and blood urea in Group 2 were $7.1 \pm 1.9 \mathrm{mg} / \mathrm{dl}$ and $123.9 \pm 53.4 \mathrm{mg} / \mathrm{dl}$, respectively. The prevalence of peripheral neuropathy was $60 \%$ based on clinical symptoms and $92 \%$ based on electrophysiological parameters.

Among 100 patients in Group 2, 70 patients (70\%) had definite damage, 22 patients $(22 \%)$ had early damage, and 8 patients $(8 \%)$ had no evidence of significant peripheral neuropathy. In diabetic subgroup, definite damage was seen in $47(94 \%)$ patients and $3(6 \%)$ patients had no significant peripheral neuropathy. In nondiabetic subgroup $(n=50)$, definite damage was 
Table 5: Comparison of electrophysiological parameters of 200 predialysis chronic kidney disease patients who participated in the study

\begin{tabular}{|c|c|c|c|c|}
\hline \multirow{3}{*}{$\begin{array}{l}\text { Electrophysiological } \\
\text { parameters }\end{array}$} & \multicolumn{3}{|c|}{ Mean \pm SD } & \multirow[t]{3}{*}{$P$} \\
\hline & \multirow{2}{*}{$\begin{array}{l}\text { Predialysis patients } \\
\quad(n=200), n(\%)\end{array}$} & \multicolumn{2}{|c|}{ Serum creatinine } & \\
\hline & & $2-4.9 \mathrm{mg} / \mathrm{dl}(n=100), n(\%)$ & $\geq 5 \mathrm{mg} / \mathrm{dl}(n=100), n(\%)$ & \\
\hline \multicolumn{5}{|l|}{ Median nerve } \\
\hline $\mathrm{dL}(\mathrm{ms})$ & $3.8 \pm 0.8$ & $3.7 \pm 0.7$ & $3.8 \pm 0.8$ & 0.237 \\
\hline $\mathrm{CV}(\mathrm{m} / \mathrm{s})$ & $51.4 \pm 4.7$ & $52.3 \pm 4.9$ & $50.5 \pm 4.4$ & 0.025 \\
\hline Amplitude (mV) & $6.9 \pm 2.7$ & $7.6 \pm 2.8$ & $6.1 \pm 2.3$ & 0.004 \\
\hline \multicolumn{5}{|l|}{ F wave } \\
\hline Normal & $98(49)$ & $52(52)$ & $46(46)$ & 0.471 \\
\hline Prolonged & $100(50)$ & $46(46)$ & $54(54)$ & \\
\hline Absent & $2(1)$ & $2(2)$ & 0 & \\
\hline \multicolumn{5}{|l|}{ Ulnar nerve } \\
\hline $\mathrm{dL}(\mathrm{ms})$ & $2.9 \pm 2.1$ & $3.1 \pm 2.9$ & $2.9 \pm 0.6$ & 0.159 \\
\hline $\mathrm{CV}(\mathrm{m} / \mathrm{s})$ & $52.3 \pm 5.4$ & $53.5 \pm 5.5$ & $51.2 \pm 5.1$ & 0.012 \\
\hline Amplitude (mV) & $6.8 \pm 2.2$ & $6.8 \pm 2.1$ & $6.7 \pm 2.3$ & 0.328 \\
\hline \multicolumn{5}{|l|}{ F wave } \\
\hline Normal & $82(41)$ & $52(52)$ & $30(30)$ & 0.079 \\
\hline Prolonged & $112(56)$ & $46(46)$ & $66(66)$ & \\
\hline Absent & $6(3)$ & $2(2)$ & $4(4)$ & \\
\hline \multicolumn{5}{|l|}{ CP nerve } \\
\hline $\mathrm{dL}(\mathrm{ms})$ & $3.5 \pm 1.4$ & $3.4 \pm 1.5$ & $3.5 \pm 1.3$ & 0.451 \\
\hline $\mathrm{CV}(\mathrm{m} / \mathrm{s})$ & $38.7 \pm 14.5$ & $38.7 \pm 15.1$ & $38.7 \pm 14.0$ & 0.624 \\
\hline Amplitude (mV) & $2.8 \pm 2.4$ & $2.7 \pm 2.3$ & $2.8 \pm 2.4$ & 0.787 \\
\hline \multicolumn{5}{|l|}{ F wave } \\
\hline Normal & $120(60)$ & $66(66)$ & $54(54)$ & 0.323 \\
\hline Prolonged & $6(3)$ & $4(4)$ & $2(2)$ & \\
\hline Absent & $74(37)$ & $30(30)$ & $44(44)$ & \\
\hline \multicolumn{5}{|l|}{ Posterior tibial nerve } \\
\hline $\mathrm{dL}(\mathrm{ms})$ & $3.7 \pm 1.4$ & $3.7 \pm 1.1$ & $3.7 \pm 1.7$ & 0.861 \\
\hline $\mathrm{CV}(\mathrm{m} / \mathrm{s})$ & $39.2 \pm 12.9$ & $40.3 \pm 12.1$ & $38.2 \pm 13.9$ & 0.470 \\
\hline Amplitude (mV) & $5.9 \pm 4.5$ & $5.9 \pm 4.3$ & $5.9 \pm 4.8$ & 0.750 \\
\hline \multicolumn{5}{|l|}{ F wave } \\
\hline Normal & $116(58)$ & $64(64)$ & $52(52)$ & 0.324 \\
\hline Prolonged & $40(20)$ & $20(20)$ & $20(20)$ & \\
\hline Absent & $44(22)$ & $16(16)$ & $28(28)$ & \\
\hline \multicolumn{5}{|l|}{ Median nerve sensory } \\
\hline dL (ms) & $2.7 \pm 0.9$ & $2.7 \pm 0.7$ & $2.7 \pm 1.0$ & 0.505 \\
\hline $\mathrm{CV}(\mathrm{m} / \mathrm{s})$ & $43.7 \pm 13.3$ & $45.9 \pm 11.2$ & $41.4 \pm 14.9$ & 0.072 \\
\hline Amplitude (mV) & $11.4 \pm 9.1$ & $14.2 \pm 10.2$ & $8.6 \pm 6.9$ & 0.003 \\
\hline \multicolumn{5}{|l|}{ Ulnar nerve sensory } \\
\hline $\mathrm{dL}(\mathrm{ms})$ & $1.9 \pm 0.9$ & $2.1 \pm 0.7$ & $1.7 \pm 1.1$ & 0.268 \\
\hline $\mathrm{CV}(\mathrm{m} / \mathrm{s})$ & $39.2 \pm 19.7$ & $45.3 \pm 14.6$ & $33.1 \pm 22.1$ & 0.003 \\
\hline Amplitude (mV) & $7.8 \pm 7.4$ & $9.9 \pm 8.0$ & $5.7 \pm 6.0$ & 0.001 \\
\hline \multicolumn{5}{|l|}{ Sural nerve sensory } \\
\hline $\mathrm{dL}(\mathrm{ms})$ & $1.6 \pm 1.4$ & $1.9 \pm 1.3$ & $1.4 \pm 1.4$ & 0.139 \\
\hline $\mathrm{CV}(\mathrm{m} / \mathrm{s})$ & $29.4 \pm 24.5$ & $33.5 \pm 23.9$ & $25.3 \pm 24.7$ & 0.151 \\
\hline Amplitude (mV) & $5.4 \pm 6.9$ & $5.5 \pm 6.5$ & $5.2 \pm 7.4$ & 0.270 \\
\hline Duration of CKD (years) & $3.4 \pm 4.2$ & $2.8 \pm 1.8$ & $3.9 \pm 5.7$ & 0.201 \\
\hline
\end{tabular}

CKD: Chronic kidney disease, dL: Distal latency, CV: Conduction velocity; SD: Standard deviation, CP: Common peroneal

seen in $23(46 \%)$ patients, early damage was seen in $22(44 \%)$ patients, and $5(10 \%)$ patients had no significant peripheral neuropathy. The most common patterns of peripheral neuropathy were pure axonal sensorimotor neuropathy $(52 \%)$ followed by mixed sensorimotor neuropathy pattern $(30 \%)$.

There was no statistically significant difference on comparing symptoms and signs of two subgroups of Group 2. 
On comparing electrophysiological data between two subgroups of Group 2, diabetic subgroup showed statistically significant prolonged median nerve motor distal latency $(P=0.004)$, low median nerve motor conduction velocity $(P=0.004)$, low median nerve motor amplitude $(P=0.022)$, prolonged median nerve F wave $(P=0.022)$, prolonged ulnar nerve motor distal latency $(P=0.007)$, low ulnar nerve motor conduction velocity $(P=0.001)$, prolonged ulnar nerve motor F wave $(P=0.002)$, low common peroneal nerve motor conduction velocity $(P=0.001)$, low common peroneal nerve motor amplitude $(P<0.001)$, absent common peroneal nerve $\mathrm{F}$ wave $(P<0.001)$, low posterior tibial nerve motor conduction velocity $(P<0.001)$, low posterior tibial nerve motor amplitude $(P=0.001)$, absent posterior tibial $\mathrm{F}$ wave $(P<0.001)$, low median nerve sensory conduction velocity $(P=0.005)$, low median nerve sensory amplitude $(P<0.001)$, low ulnar nerve sensory amplitude $(P<0.001)$, low sural nerve sensory distal latency $(P<0.001)$, low sural nerve sensory conduction velocity $(P<0.001)$, and low sural nerve sensory amplitude $(P<0.001)$. Patients without diabetes mellitus showed statistically significant prolonged median nerve sensory distal latency $(P=0.016)$, prolonged ulnar nerve sensory distal latency $(P=0.002)$, and low ulnar nerve sensory conduction velocity $(P=0.010)$.

\section{DisCUSSION}

CKD is characterized by chronic progressive decline in renal function. Multiple neurological complications are seen in CKD, of which uremic neuropathy is most disabling. There is segmental demyelination and axonal degeneration in peripheral nerves. Management of peripheral neuropathy in CKD patients requires establishment of neuropathy and determination of type of neuropathy by NCSs. The prevalence of peripheral neuropathy was established in end-stage renal disease in various international studies, but the prevalence in predialysis patients is not well established. In the present study, we excluded patients on maintenance dialysis, to negate the beneficial effect of dialysis on peripheral neuropathy. The present study was undertaken to study the prevalence of peripheral neuropathy, clinical features, electrophysiological features, and severity of peripheral neuropathy in predialysis patients of rural South India. The present study showed a high prevalence of uremic neuropathy; $45 \%$ based on clinical symptoms and $90 \%$ according to electrophysiological studies. The prevalence of peripheral neuropathy in uremia in the present study was slightly higher when compared to other published studies $^{[18-20]}$ such as Aggarwal et al. (70\%), Sultan et al. (62.5\%), and Madhusudhana Babu et al. (65\%).
About $45 \%$ patients had asymptomatic peripheral neuropathy in the present study. The prevalence of asymptomatic peripheral neuropathy was more common in mild-to-moderate renal failure group than severe renal failure group (58\% vs. $32 \%)$. This further strengthens the need for regular NCSs for early diagnosis and appropriate management of peripheral neuropathy in predialysis patients.

In the present study, positive sensory symptoms were seen in $40 \%$ and negative sensory symptoms were seen in $35 \%$ of patients, which were more common in severe renal failure group when compared with mild-to-moderate renal failure group. Autonomic symptoms were seen in $8(8 \%)$ patients, which were seen only in severe renal failure group of patients. Symptomatic neuropathy was seen in $51 \%$ of predialysis CKD patients according to Aggarwal et al., ${ }^{[18]}$ which was $45 \%$ in the present study. According to Krishnan et al. ${ }^{[13]}$ and Laaksonen et al., ${ }^{[21]}$ symptomatic peripheral neuropathy was seen in $91 \%$ and $62 \%$ of CKD patients, respectively.

\section{Electrophysiological parameters in predialysis chronic kidney disease patients}

Comparison of electrophysiological parameters in 200 predialysis CKD patients with other published international studies ${ }^{[19,22]}$ is shown in Table 6. Data regarding electrophysiological parameters in predialysis CKD patients are sparse in Indian literature.

On comparing electrophysiological parameters of predialysis patients, motor distal latency and motor conduction velocity were similar to other published studies, but motor amplitudes were low in the present study. Sample size in the present study was large, when compared to other published studies.

Most common nerves involved in the present study were sural nerve, median sensory nerve, and ulnar sensory nerve. Most common motor nerves involved in the present study were common peroneal nerve, posterior tibial nerve followed by median nerve, and ulnar nerve. Lower limbs were most commonly affected than upper limbs, which indicate a length dependent pattern of peripheral neuropathy. As length-dependent pattern of neuropathy is seen in uremic neuropathy, lower limbs are involved early in CKD. Upper limb nerves are later involved as renal failure worsens theoretically. Similar pattern was seen in the present study also. Sensory nerves were commonly affected than motor nerves in the present study. These results were similar to other published international studies. ${ }^{[19,22]}$

According to Aggarwal et al., ${ }^{[18]}$ mean nerve conduction velocities $(\mathrm{m} / \mathrm{s})$ of right median, ulnar, common 


\begin{tabular}{|c|c|c|c|}
\hline \multicolumn{4}{|c|}{$\begin{array}{l}\text { Table 6: Comparison of electrophysiological parameters } \\
\text { in predialysis patients as reported in published studies }\end{array}$} \\
\hline Variable & Sultan $^{[19]}$ & Fatima $^{[22]}$ & $\begin{array}{c}\text { Present } \\
\text { study }\end{array}$ \\
\hline Country & Egypt & Pakistan & India \\
\hline Sample size & 20 & 30 & 200 \\
\hline \multicolumn{4}{|l|}{$\begin{array}{l}\text { Electrophysiological } \\
\text { parameters }\end{array}$} \\
\hline \multicolumn{4}{|l|}{ Median nerve } \\
\hline $\mathrm{dL}(\mathrm{ms})$ & $3.1 \pm 0.6$ & $8.3 \pm 0.2$ & $3.8 \pm 0.8$ \\
\hline $\mathrm{CV}(\mathrm{m} / \mathrm{s})$ & $55.6 \pm 6.8$ & $53.7 \pm 2.2$ & $51.4 \pm 4.7$ \\
\hline Amplitude (mV) & $12.7 \pm 5.5$ & $13.2 \pm 0.4$ & $6.9 \pm 2.7$ \\
\hline \multicolumn{4}{|l|}{ Ulnar nerve } \\
\hline $\mathrm{dL}(\mathrm{ms})$ & $2.6 \pm 0.5$ & NA & $2.9 \pm 2.1$ \\
\hline $\mathrm{CV}(\mathrm{m} / \mathrm{s})$ & $57.7 \pm 6.5$ & NA & $52.3 \pm 5.4$ \\
\hline Amplitude (mV) & $13.8 \pm 3.5$ & NA & $6.8 \pm 2.2$ \\
\hline \multicolumn{4}{|l|}{ CP nerve } \\
\hline $\mathrm{dL}(\mathrm{ms})$ & $5.4 \pm 1.3$ & $9.4 \pm 0.1$ & $3.5 \pm 1.4$ \\
\hline $\mathrm{CV}(\mathrm{m} / \mathrm{s})$ & $43.5 \pm 4.2$ & $56.3 \pm 1.7$ & $38.7 \pm 14.5$ \\
\hline Amplitude (mV) & $3.8 \pm 2.5$ & $4.5 \pm 1.1$ & $2.8 \pm 2.4$ \\
\hline \multicolumn{4}{|l|}{ Posterior tibial nerve } \\
\hline $\mathrm{dL}(\mathrm{ms})$ & $5.1 \pm 1.0$ & NA & $3.7 \pm 1.4$ \\
\hline $\mathrm{CV}(\mathrm{m} / \mathrm{s})$ & $42.9 \pm 5.4$ & NA & $39.2 \pm 12.9$ \\
\hline Amplitude (mV) & $12.4 \pm 5.0$ & NA & $5.9 \pm 4.5$ \\
\hline \multicolumn{4}{|c|}{ Median nerve sensory } \\
\hline $\mathrm{dL}(\mathrm{ms})$ & NA & NA & $2.7 \pm 0.9$ \\
\hline $\mathrm{CV}(\mathrm{m} / \mathrm{s})$ & NA & NA & $43.7 \pm 13.3$ \\
\hline Amplitude (mV) & NA & NA & $11.4 \pm 9.1$ \\
\hline \multicolumn{4}{|l|}{ Ulnar nerve sensory } \\
\hline $\mathrm{dL}(\mathrm{ms})$ & $2.9 \pm 0.3$ & NA & $1.9 \pm 0.9$ \\
\hline $\mathrm{CV}(\mathrm{m} / \mathrm{s})$ & $54.5 \pm 5.4$ & NA & $39.2 \pm 19.7$ \\
\hline Amplitude (mV) & $14.0 \pm 16.3$ & NA & $7.8 \pm 7.4$ \\
\hline \multicolumn{4}{|l|}{ Sural nerve sensory } \\
\hline $\mathrm{dL}(\mathrm{ms})$ & $3.9 \pm 0.8$ & NA & $1.6 \pm 1.4$ \\
\hline $\mathrm{CV}(\mathrm{m} / \mathrm{s})$ & $39.9 \pm 5.4$ & NA & $29.4 \pm 24.5$ \\
\hline Amplitude (mV) & $9.8 \pm 3.8$ & NA & $5.4 \pm 6.9$ \\
\hline
\end{tabular}

dL: Distal latency, CV: Conduction velocity, NA: Not applicable,

CP: Common peroneal

peroneal, and posterior tibial nerves were $51.34 \pm 6.07$, $53.04 \pm 5.91,44.72 \pm 6.14$, and $44.2 \pm 5.17$, respectively, in predialysis patients, which were almost similar to the present study. In predialysis patients $(n=200)$, mononeuropathy was seen in 6 patients in the form of carpal tunnel syndrome.

Ulnar nerve sensory amplitude and conduction velocity, median nerve sensory amplitude, median nerve motor amplitude, and conduction velocity were statistically significant electrophysiological parameters to differentiate severe renal failure group of patients from mild-to-moderate renal failure group of patients.

The prevalence of peripheral neuropathy was $88 \%$ in mild-to-moderate renal failure group and $92 \%$ in severe renal failure group. This showed that the prevalence of peripheral neuropathy increases as renal failure worsens.
In addition, severity of peripheral neuropathy was also more in severe renal failure group of patients which was similar to other Indian study by Aggarwal et al..$^{[18]}$

Diabetic CKD patients showed more severe and high prevalence of peripheral neuropathy when compared to nondiabetic CKD patients $(97 \%$ vs. $83 \%)$, which was almost similar in both Group 1 and Group 2. As peripheral neuropathy depends on blood sugar level in addition to uremia, diabetic CKD patients showed higher prevalence and severity of peripheral neuropathy.

In the present study, statistically most significant electrophysiological parameters differentiating diabetics and nondiabetics in mild-to-moderate renal failure group were median nerve motor distal latency, ulnar nerve motor conduction velocity, ulnar nerve $\mathrm{F}$ wave, common peroneal nerve motor conduction velocity, posterior tibial nerve motor conduction velocity, and ulnar nerve sensory amplitude. Statistically most significant electrophysiological parameters differentiating diabetics and nondiabetics in severe renal failure group were common peroneal nerve $\mathrm{F}$ wave, posterior tibial $\mathrm{F}$ wave, median nerve sensory amplitude, ulnar nerve sensory amplitude, sural nerve sensory distal latency, sural nerve sensory conduction velocity, sural nerve sensory amplitude, common peroneal nerve motor conduction velocity, common peroneal nerve motor amplitude, and ulnar nerve motor conduction velocity. Indian literature comparing diabetic CKD patients and nondiabetic CKD patients is sparse.

Pure axonal sensorimotor neuropathy and mixed sensorimotor neuropathy were most common patterns in predialysis CKD patients.

\section{Limitations of the present study}

1. This study is a hospital-based study, so it is not an accurate measure of community-based prevalence of neuropathy in predialysis CKD patients.

2. Electrophysiologic parameters might be slightly altered due to the presence of edema in CKD patients.

\section{Conclusion}

Peripheral neuropathy is common in predialysis CKD patients, prevalence and severity of which increases as renal failure worsens. Predialysis patients with diabetes show a higher prevalence of peripheral neuropathy when compared with nondiabetics, with more severity in severe renal failure. Pure axonal sensorimotor neuropathy and mixed sensorimotor neuropathy are most common patterns in predialysis CKD patients. Early diagnosis of peripheral neuropathy in CKD patients by NCSs is required to minimize patient discomfort. 


\section{Financial support and sponsorship}

Nil.

\section{Conflicts of interest}

There are no conflicts of interest.

\section{REFERENCES}

1. KDOQI; National Kidney Foundation. KDOQI clinical practice guidelines and clinical practice recommendations for anemia in chronic kidney disease. Am J Kidney Dis 2006;47 5 Suppl 3:S11-145.

2. Levey AS, Eckardt KU, Tsukamoto Y, Levin A, Coresh J, Rossert $\mathrm{J}$, et al. Definition and classification of chronic kidney disease: A position statement from kidney disease: Improving global outcomes (KDIGO). Kidney Int 2005;67:2089-100.

3. National Kidney Foundation. K/DOQI clinical practice guidelines for chronic kidney disease: Evaluation, classification, and stratification. Am J Kidney Dis 2002;39:S1-266.

4. Meyer TW, Hostetter TH. Uremia. N Engl J Med 2007;357:1316-25.

5. Brouns R, De Deyn PP. Neurological complications in renal failure: A review. Clin Neurol Neurosurg 2004;107:1-16.

6. Krishnan AV, Kiernan MC. Neurological complications of chronic kidney disease. Nat Rev Neurol 2009;5:542-51.

7. Asbury AK, Victor M, Adams RD. Uremic polyneuropathy. Arch Neurol 1963;8:413-28.

8. Dyck PJ, Johnson WJ, Lambert EH, O'Brien PC. Segmental demyelination secondary to axonal degeneration in uremic neuropathy. Mayo Clin Proc 1971;46:400-31.

9. Nielsen VK, Winkel P. The peripheral nerve function in chronic renal failure 3. A multivariate statistical analysis of factors presumed to affect the development of clinical neuropathy. Acta Med Scand 1971;190:119-25.

10. Bolton CF, Baltzan MA, Baltzan RB. Effects of renal transplantation on uremic neuropathy. A clinical and electrophysiologic study. N Engl J Med 1971;284:1170-5.

11. Nielsen VK. The peripheral nerve function in chronic renal failure. II. Intercorrelation of clinical symptoms and signs and clinical grading of neuropathy. Acta Med Scand 1971;190:113-7.

12. Halar EM, Brozovich FV, Milutinovic J, Inouye VL, Becker VM H-reflex latency in uremic neuropathy: Correlation with NCV and clinical findings. Arch Phys Med Rehabil 1979;60:174-7.

13. Krishnan AV, Phoon RK, Pussell BA, Charlesworth JA, Bostock H, Kiernan MC, et al. Altered motor nerve excitability in end-stage kidney disease. Brain 2005;128:2164-74.

14. Misulis KE, Head TC. Nerve conduction study and electromyography. In: Pioli SF, editors. Essentials of clinical neurophysiology. $3^{\text {rd }}$ ed. Burlington: Buerworth Heinemann 2003:129-44.

15. Chen S, Andary M, Buschbacher R, Del Toro D, Smith B, So $\mathrm{Y}$, et al. Electrodiagnostic reference values for upper and lower limb nerve conduction studies in adult populations. Muscle Nerve 2016;54:371-7.

16. Nobrega JA, Pinheiro DS, Manzano GM, Kimura J. Various aspects of F-wave values in a healthy population. Clin Neurophysiol 2004;115:2336-42.

17. Bolton CF, Carter KM. Human sensory nerve compound action potential amplitude: Variation with sex and finger circumference. J Neurol Neurosurg Psychiatry 1980;43:925-8.

18. Aggarwal HK, Sood S, Jain D, Kaverappa V, Yadav S. Evaluation of spectrum of peripheral neuropathy in predialysis patients with chronic kidney disease. Ren Fail 2013;35:1323-9.

19. Sultan LI. Evaluation of the clinical and neurophysiologic parameters of peripheral nerve functions in uremic Egyptian patients. Egypt J Neurol Psychiatr Neurosurg 2007;44:473-87.

20. Madhusudhana Babu M, Ravi Kiran M, Ravindra K, Srinivas V, Kandregula P, Vikram Vardhan R. Clinical manifestations and prevalence of peripheral neuropathy and nerve dysfunction in patients with chronic kidney disease. Int J Res Med Sci 2015;3:451-5.

21. Laaksonen S, Metsärinne K, Voipio-Pulkki LM, Falck B. Neurophysiologic parameters and symptoms in chronic renal failure. Muscle Nerve 2002;25:884-90.

22. Fatima K, Majid A. Motor nerve conduction studies in patients with chronic renal failure. J Rawalpindi Med Coll 2010;14:11-4. 